\title{
One Pot Synthesis of Some New Pyrazole Containing Octahydroquinazolinone Derivatives Catalyzed by p-Toluenesulfonic Acid in Water
}

\author{
SUNEEL SAHU* and S P. SHRIVASTAVA \\ Synthetic Organic Chemistry Laboratory, Department of Chemistry, \\ Dr. Harisingh Gour Vishwvidhyalaya, Sagar (M.P.)-470003, India \\ sahusuneel6@gmail.com
}

Receivd 17 March 2016/ Accepted 14 April 2016

\begin{abstract}
A novel green and efficient one-pot three component reaction synthesis of octahydroquinazolinone compounds (4a-1) in good yield has been reported. The methodology initially involved the formation of targeted compounds via reaction of a variety of pyrazolo carbaldehydes with dimedone and urea /thiourea in the presence of catalytic amount of $p$-TsOH in water. The structures of synthesized compounds were confirmed by spectraldata.
\end{abstract}

Keywords: Octahydroquinazolinone, Pyrazole, Multicomponent

\section{Introduction}

Pyrazole and quinazolinone containing molecules are of particular interest, especially in the field of medicinal chemistry ${ }^{1}$ For example, several classes of agrochemicals ${ }^{2,3}$ and pharmaceuticals $^{4-6}$ discovered and identified. Quinazolinone derivatives have attracted considerable attention since they exhibit potent antibacterial activity ${ }^{7,8}$ Synthesis of quinazolinone derivatives have been developed several methods. Multicomponent reactions (MCRs) have emerged as a powerful tool in heterocyclic synthesis ${ }^{9,10}$. There are few report synthesis of octahydroquinazolinone derivatives with aromatic aldehydes using catalysts such as conc. $\mathrm{H}_{2} \mathrm{SO}_{4}{ }^{9} \mathrm{NH}_{4} \mathrm{VO}_{3}{ }^{10}$ and ionic liquid ${ }^{11,12}$ in multicomponent reaction ${ }^{13}$. Octahydroquinazolinone derivatives are synthesized in absolute ethanol but with low yields of products $(19-69 \%)^{8}$ So the development of new carbaldehydes derivative with a environmentally friendly and high- yielding, green approach in the reaction. $\mathrm{TsOH}^{14}$ has facile and eco friendly catalyst. We use the para-Toluenesulphonilic acid ( $p$-TsOH) as an organic acid catalyst and water as a solvent select the bases of economic and easily availability will makes this reaction eco friendly. These views introduce the Synthesis of some new Octahydroquinazolinone derivatives. So we used water as a solvent and $p$-TsOH as a catalyst in the one pot reaction of dimedone (1), urea/ thiourea (2) and pyrazole containing carbaldehyde ${ }^{15,16}(\mathbf{3 a - f})$. 


\section{Experimental}

The completion of reactions of prepare compounds were checked by thin-layer chromatography (TLC) on aluminum plates coated with silica gel $60 \mathrm{~F}_{254}, 0.25 \mathrm{~mm}$ thickness (Merck). Separation of compounds was carried out by column chromatography using silica gel (100-200 mesh). Melting points were determined by open capillary method using a melting point apparatus Buchi Melting Point B-540 apparatus and are uncorrected. IR spectra were recorded with $\mathrm{KBr}$ discs (for solids), with a Shimadzu FTIR-8400S instrument and are expressed in $\mathrm{cm}^{-1}$. NMR spectra were recorded at $25.0{ }^{\circ} \mathrm{C}$ with a Bruker Avance III 400 spectrometer operating at $400 \mathrm{MHz}$ for ${ }^{1} \mathrm{H}$ and $100 \mathrm{MHz}$ for ${ }^{13} \mathrm{C}$ instrument DMSO- $\mathrm{d}^{6}$ using as solvent and tetramethylsilane (TMS) as the internal standard $(0.00 \mathrm{ppm})$. Chemical shifts values were given in $\delta$ (ppm) scales.

\section{Synthesis}

Synthesis of 7,7-dimethyl-4-(3-phenyl-1H-pyrazol-4-yl)-3,4,7,8-tetra hydroquinazoline-2,5(1H,6H)-dione

The reaction of carbaldehyde $(0.01 \mathrm{~mol}), 5,5$-dimethyl-1,3-cyclohexanedione $(0.01 \mathrm{~mol})$, and thiourea $(0.015 \mathrm{~mol})$ in the presence of $p$-TsOH $(5 \mathrm{mmol} \%)$ was found to be complete within $2 \mathrm{~h}$, as indicated by TLC, in refluxing water $\left(\mathrm{H}_{2} \mathrm{O} 20 \mathrm{~mL}\right)$ and 4-phenyl-7,7dimethyl-5-oxo-1,2,3,4,5,6,7,8-octahydroquinazoline-2-thione 4a in $92 \%$ yield precipitated from the reaction mixture on cooling to room temperature. Precipitate was filtered and recrystallize with ethanol (Scheme1).

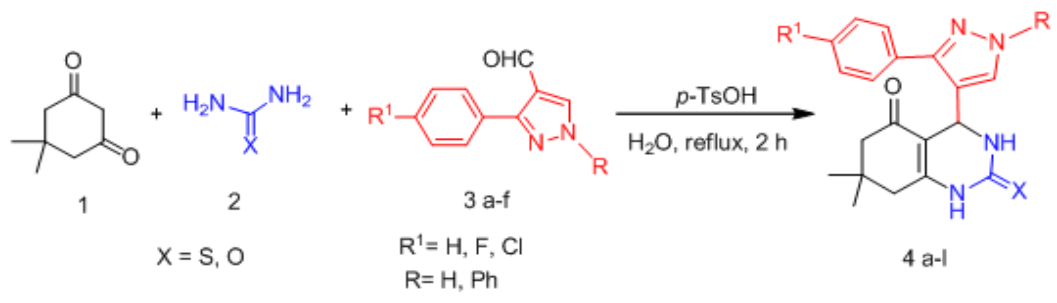

\section{Scheme 1}

\section{Results and Discussion}

7,7-Dimethyl-4-(3-phenyl-1H-pyrazol-4-yl)-3,4,7,8-tetrahydro (1H,6H)-dione (4a)

quinazoline-2,5

Milky White solid, 92\% yield, mp-203 ${ }^{\circ} \mathrm{C}$; IR (KBr):3214,17321678 ${ }^{1} \mathrm{H}$ NMR (DMSO$\left.\mathrm{d}_{6}, 400 \mathrm{MHz}\right) \delta 7.67(\mathrm{~d}, 2 \mathrm{H} \mathrm{J}=5.0 \mathrm{~Hz}) \delta 7.34(\mathrm{~s}, 1 \mathrm{H}) \delta 7.02-6.94(\mathrm{~m}, 5 \mathrm{H}) \delta 5.59(\mathrm{~s}, 1 \mathrm{H}) \delta 4.78$ $(\mathrm{s}, 1 \mathrm{H}) \delta 2.26-2.29(\mathrm{~d}, 2 \mathrm{H} \mathrm{J}=16 \mathrm{~Hz}) \delta 1.06(\mathrm{~s}, 3 \mathrm{H}) \delta 0.96(\mathrm{~s}, 3 \mathrm{H}){ }^{13} \mathrm{C} \mathrm{NMR}(100 \mathrm{MHz}): \delta 196.76$, $179.65,136.78,132.15,131.11,129.31,128.79,128.41,117.34,115.62,33.89,32.41$, 27.39, $23.21 \mathrm{~ms}(\mathrm{ESI}+)$ : $m / z: 336.29[\mathrm{M}+\mathrm{H}]+$ Mol.Formula: $\mathrm{C}_{19} \mathrm{H}_{20} \mathrm{~N}_{4} \mathrm{O}_{2}$.

4-(3-(4-Fluorophenyl)-1H-pyrazol-4-yl)-7,7-dimethyl-3,4,7,8-tetra hydroquinazoline $-2,5(1 H, 6 H)$-dione $(4 b)$

Milky White solid, 90\% yield, mp-215 ${ }^{\circ} \mathrm{C}$; IR(KBr): 3230, 1743, 1650, ${ }^{1} \mathrm{H}$ NMR (DMSO$\left.\mathrm{d}_{6}, 400 \mathrm{MHz}\right) \delta 7.89(\mathrm{~d}, 2 \mathrm{H} \mathrm{J}=8.0 \mathrm{~Hz}) \delta 7.46(\mathrm{~s}, 1 \mathrm{H}) \delta 7.11-7.02(\mathrm{~m}, 4 \mathrm{H}) \delta 5.93(\mathrm{~s}, 1 \mathrm{H}) \delta 4.89(\mathrm{~s}, 1 \mathrm{H})$ $\delta 2.31-2.35(\mathrm{~d}, 2 \mathrm{H} \mathrm{J}=16 \mathrm{~Hz}) \delta 1.10(\mathrm{~s}, 3 \mathrm{H}) \quad \delta 0.99(\mathrm{~s}, 3 \mathrm{H}){ }^{13} \mathrm{C}$ NMR $(100 \mathrm{MHz}): \delta 198.64,181.64$, $146.45,142.23,141.28,136.45,133.44,130.64,128.41,117.34,115.62$, 35.89, 33.71, 30.59, 28.63, $27.35 \mathrm{~ms}(\mathrm{ESI}+)$ : $\mathrm{m} / \mathrm{z}: 354.27[\mathrm{M}+\mathrm{H}]+$. Mol. Formula: $\mathrm{C}_{19} \mathrm{H}_{19} \mathrm{FN}_{4} \mathrm{O}_{2}$. 
4-(3-(4-Chlorophenyl)-1H-pyrazol-4-yl)-7,7-dimethyl-3,4,7,8-tetra hydroquinazoline2,5(1H,6H)-dione $(4 \mathrm{c})$

Milky White solid, 95\% yield, mp-194 ${ }^{\circ} \mathrm{C}$, IR (KBr):3222,1753,1642, ${ }^{1} \mathrm{H}$ NMR (DMSO$\left.\mathrm{d}_{6}, 400 \mathrm{MHz}\right) \delta 7.72(\mathrm{~d}, 2 \mathrm{H} \mathrm{J}=10.0 \mathrm{~Hz}) \delta 7.40(\mathrm{~s}, 1 \mathrm{H}) \delta 6.95-7.02(\mathrm{~m}, 4 \mathrm{H}) \delta 5.42(\mathrm{~s}, 1 \mathrm{H}) \delta 4.70(\mathrm{~s}, 1 \mathrm{H})$ $\delta 2.27-2.30(\mathrm{~d}, 2 \mathrm{H} \quad \mathrm{J}=16 \mathrm{~Hz}) \delta 1.15(\mathrm{~s}, 3 \mathrm{H}) \quad \delta 0.93(\mathrm{~s}, 3 \mathrm{H}){ }^{13} \mathrm{C} \mathrm{NMR}(100 \mathrm{MHz}) \delta 192.14,176.26$, $142.15,141.03$, 140.67, 134.12, 133.21, 129.14, 128.17, 117.04, 114.52, 35.53, 32.43, 29.91, 29.14, $26.04 \mathrm{~ms}(\mathrm{ESI}+): \mathrm{m} / z: 370.10[\mathrm{M}+\mathrm{H}]+$. Mol. Formula: $\mathrm{C}_{19} \mathrm{H}_{19} \mathrm{ClN}_{4} \mathrm{O}_{2}$.

4-(1,3-Diphenyl-1H-pyrazol-4-yl)-7,7-dimethyl-3,4,7,8-tetrahydro quinazoline-2,5 (1H,6H)-dione (4d)

Milky White solid, 96\% yield, mp-185 ${ }^{\circ} \mathrm{C}$; IR (KBr):3257,1742,1632 ${ }^{1} \mathrm{H}$ NMR (DMSO$\left.\mathrm{d}_{6}, 400 \mathrm{MHz}\right) \delta 7.58(\mathrm{~d}, 2 \mathrm{H} \mathrm{J}=10.0 \mathrm{~Hz}) \quad \delta 6.55-6.50 \quad(\mathrm{~m}, 5 \mathrm{H}) \delta 6.43-6.36(\mathrm{~m}, 5 \mathrm{H}) \delta 5.12(\mathrm{~s}, 1 \mathrm{H})$ $\delta 4.53(\mathrm{~s}, 1 \mathrm{H}) \delta 2.07-2.11(\mathrm{~d}, 2 \mathrm{H} \mathrm{J}=16 \mathrm{~Hz}) \delta 1.03(\mathrm{~s}, 3 \mathrm{H}) \delta 0.96(\mathrm{~s}, 3 \mathrm{H}){ }^{13} \mathrm{C}$ NMR $(100 \mathrm{MHz}):$ $\delta 189.12,178.43,146.45,143.83,143.65,142.06,141.73,141.28,134.42,132.42,130.34$, $129.34,118.46,115.62,34.89,32.71,29.59,28.63,26.35, \mathrm{~ms}(\mathrm{ESI}+): \mathrm{m} / z: 412.42[\mathrm{M}+\mathrm{H}]+$. Mol.Formula: $\mathrm{C}_{25} \mathrm{H}_{24} \mathrm{~N}_{4} \mathrm{O}_{2}$.

4-(3-(4-Fluorophenyl)-1-phenyl-1H-pyrazol-4-yl)-7,7-dimethyl-3,4,7,8-tetrahydroquinazoline-2,5(1H,6H)-dione $(4 \mathrm{e})$

Milky White solid, 89\% yield, mp-202 ${ }^{\circ} \mathrm{C}$; IR(KBr): 32558, 1749,1643 ${ }^{1} \mathrm{H}$ NMR (DMSO$\left.\mathrm{d}_{6}, 400 \mathrm{MHz}\right) \delta 7.76(\mathrm{~d}, 2 \mathrm{H} \mathrm{J}=10.0 \mathrm{~Hz}) \quad \delta 6.79-6.83(\mathrm{~m}, 4 \mathrm{H}) \quad \delta 6.45-7.49(\mathrm{~m}, 5 \mathrm{H}) \delta 5.27(\mathrm{~s}, 1 \mathrm{H})$ $\delta 4.68(\mathrm{~s}, 1 \mathrm{H}) \delta 2.27-2.23(\mathrm{~d}, 2 \mathrm{H} \mathrm{J}=16 \mathrm{~Hz}) \delta 1.13(\mathrm{~s}, 3 \mathrm{H}) \delta 0.99(\mathrm{~s}, 3 \mathrm{H}){ }^{13} \mathrm{C}$ NMR $(100 \mathrm{MHz}): \delta$ $193.45,181.63,147.87,144.43,143.92143 .04,142.61,141.91,136.79,132.42,132.41$, $129.44,119.67,117.42,34.96,33.11,30.59,28.69,26.83, \mathrm{~ms}(\mathrm{ESI}+): \mathrm{m} / z: 430.05[\mathrm{M}+\mathrm{H}]+$. Mol.Formula: $\mathrm{C}_{25} \mathrm{H}_{23} \mathrm{FN}_{4} \mathrm{O}_{2}$.

4-(3-(4-chlorophenyl)-1-phenyl-1H-pyrazol-4-yl)-7,7-dimethyl-3,4,7,8-tetrahydroquinazoline-2,5(1H,6H)-dione $(\mathbf{4 f})$

Milky White solid, 92\% yield, mp-189 ${ }^{\circ} \mathrm{C}$; IR (KBr): 3257,1742,1632 ${ }^{1} \mathrm{H}$ NMR (DMSO$\left.\mathrm{d}_{36}, 400 \mathrm{MHz}\right) \delta 7.62(\mathrm{~d}, 2 \mathrm{H} \mathrm{J}=8.0 \mathrm{~Hz}) \delta 6.67-6.72(\mathrm{~m}, 4 \mathrm{H}) \delta 6.52-7.57(\mathrm{~m}, 5 \mathrm{H}) \delta 5.05(\mathrm{~s}, 1 \mathrm{H})$ $\delta 4.65(\mathrm{~s}, 1 \mathrm{H}) \delta 2.17-2.21(\mathrm{~d}, 2 \mathrm{H} \mathrm{J}=16 \mathrm{~Hz}) \delta 1.11(\mathrm{~s}, 3 \mathrm{H}) \delta 0.98(\mathrm{~s}, 3 \mathrm{H}){ }^{13} \mathrm{C}$ NMR $(100 \mathrm{MHz}): \delta$ 192.52, 179.32, 145.72, 143.57, 143.11, 142.80, 142.23, 141.54, 134.95, 131.22, 130.81, $129.12,120.38,117.21,33.66,32.64,28.49,27.37,26.63, \mathrm{~ms}(\mathrm{ESI}+): \mathrm{m} / z: 446.03[\mathrm{M}+\mathrm{H}]+$. Mol.Formula: $\mathrm{C}_{25} \mathrm{H}_{24} \mathrm{ClN}_{4} \mathrm{O}_{2}$.

7,7-Dimethyl-4-(3-phenyl-1H-pyrazol-4-yl)-2-thioxo-1,2,3,4,7,8-hexahydroquinazolin -5(6H)-one (4g)

Milky White solid, 92\% yield, mp-201 ${ }^{\circ} \mathrm{C}$; IR (KBr): 3218,1763,1642, ${ }^{1} \mathrm{H}$ NMR (DMSO$\left.\mathrm{d}_{6}, 400 \mathrm{MHz}\right) \delta 7.71(\mathrm{~d}, 2 \mathrm{H}, \mathrm{J}=8.0 \mathrm{~Hz}) \delta 7.36(\mathrm{~s}, 1 \mathrm{H}) \delta 7.15-7.01(\mathrm{~m}, 5 \mathrm{H}) \delta 5.67(\mathrm{~s}, 1 \mathrm{H}) \delta 4.81(\mathrm{~s}, 1 \mathrm{H}$ ) $\delta 2.28-2.32(\mathrm{~d}, 2 \mathrm{H}, \mathrm{J}=16 \mathrm{~Hz}) \delta 1.11(\mathrm{~s}, 3 \mathrm{H}) \delta 0.98(\mathrm{~s}, 3 \mathrm{H}){ }^{13} \mathrm{C} \mathrm{NMR}\left(\mathrm{CDCl}_{3}, 100 \mathrm{MHz}\right): \delta$ $197.19,178.85,138.78,137.18,132.62,129.81,129.59,128.84,118.15,115.82,34.39$, $31.89,29.53,27.71, \mathrm{~ms}(\mathrm{ESI}+): \mathrm{m} / z: 352.64[\mathrm{M}+\mathrm{H}]+$. Mol.Formula: $\mathrm{C}_{19} \mathrm{H}_{20} \mathrm{~N}_{4} \mathrm{OS}$.

4-(3-(4-Fluorophenyl)-1H-pyrazol-4-yl)-7,7-dimethyl-2-thioxo-1,2,3,4,7,8hexahydroquinazolin-5(6H)-one (4h)

Milky White solid, $89 \%$ yield, mp-205 ${ }^{\circ}$; IR (KBr): 3269,1762,1647, ${ }^{1} \mathrm{H}$ NMR (DMSO$\left.\mathrm{d}_{6}, 400 \mathrm{MHz}\right) \delta 7.90(\mathrm{~d}, 2 \mathrm{H} \mathrm{J}=8.0 \mathrm{~Hz}) \delta 7.50(\mathrm{~s}, 1 \mathrm{H}) \delta 7.17-7.23(\mathrm{~m}, 4 \mathrm{H}) \delta 5.96(\mathrm{~s}, 1 \mathrm{H}) \delta 4.89(\mathrm{~s}, 1 \mathrm{H})$ 
$\delta 2.36-2.39(\mathrm{~d}, 2 \mathrm{H} \mathrm{J}=12 \mathrm{~Hz}) \delta 1.13(\mathrm{~s}, 3 \mathrm{H}) \quad \delta 0.99(\mathrm{~s}, 3 \mathrm{H}){ }^{13} \mathrm{C}$ NMR $(100 \mathrm{MHz}): \delta 198.64,182$. $64,146.45,142.23,141.28,136.45,133.44,130.64,128.41,124.96,120.51,36.83,32.98$, 31.45, 29.36, $27.46 \mathrm{~ms}(\mathrm{ESI}+): \mathrm{m} / \mathrm{z}: 370.31[\mathrm{M}+\mathrm{H}]+$. Mol. Formula: $\mathrm{C}_{19} \mathrm{H}_{19} \mathrm{FN}_{4} \mathrm{OS}$.

4-(3-(4-Chlorophenyl)-1H-pyrazol-4-yl)-7,7-dimethyl-2-thioxo-1,2,3,4,7,8hexahydroquinazolin-5(6H)-one $(4 \mathrm{i})$

Milky White solid, 94\% yield, mp-182 ${ }^{\circ} \mathrm{C}$; IR (KBr): 3254,1761,1632 ${ }^{1} \mathrm{H}$ NMR(DMSO$\left.\mathrm{d}_{6}, 400 \quad \mathrm{MHz}\right) \quad \delta 7.70(\mathrm{~d}, 2 \mathrm{H}, \quad \mathrm{J}=12.0 \mathrm{~Hz}) \quad \delta 7.42 \quad(\mathrm{~s}, 1 \mathrm{H}) \quad \delta 6.98-7.05 \quad(\mathrm{~m}, 4 \mathrm{H}) \quad \delta 5.44 \quad(\mathrm{~s}, 1 \mathrm{H})$ $\delta 4.76(\mathrm{~s}, 1 \mathrm{H}) \delta 2.28-2.32(\mathrm{~d}, 2 \mathrm{H} \mathrm{J}=16 \mathrm{~Hz}) \delta 1.12(\mathrm{~s}, 3 \mathrm{H}) \delta 0.98(\mathrm{~s}, 3 \mathrm{H}){ }^{13} \mathrm{C}$ NMR $(100 \mathrm{MHz})$ : $\delta 197.87,181.33,147.76,143.47,143.52,141.89,141.43,140.34,134.53,124.96,120.51$, 36.83, 32.98, 29.45, 27.36, 25.46, $\mathrm{ms}(\mathrm{ESI}+): \mathrm{m} / \mathrm{z}: 386.72[\mathrm{M}+\mathrm{H}]+$. Mol. Formula: $\mathrm{C}_{19} \mathrm{H}_{19} \mathrm{ClN}_{4} \mathrm{OS}$.

4-(1,3-Diphenyl-1H-pyrazol-4-yl)-7,7-dimethyl-2-thioxo-1,2,3,4,7,8-hexahydroquinazolin-5(6H)-one $(4 j)$

Milky White solid, 95\% yield, mp-196 ${ }^{\circ} \mathrm{C}$; IR (KBr): 3261,1726,1644 ${ }^{1} \mathrm{H}$ NMR (DMSO$\left.\mathrm{d}_{6}, 400 \mathrm{MHz}\right) \delta 7.60(\mathrm{~d}, 2 \mathrm{H} \mathrm{J}=10.0 \mathrm{~Hz}) \delta 6.57-6.65(\mathrm{~m}, 5 \mathrm{H}) \delta 6.40-6.33(\mathrm{~m}, 5 \mathrm{H}) \delta 5.25(\mathrm{~s}, 1 \mathrm{H})$ $\delta 4.59(\mathrm{~s}, 1 \mathrm{H}) \delta 2.11-2.14(\mathrm{~d}, 2 \mathrm{H}, \mathrm{J}=12 \mathrm{~Hz}) \delta 1.04(\mathrm{~s}, 3 \mathrm{H}) \delta 0.98(\mathrm{~s}, 3 \mathrm{H}){ }^{13} \mathrm{C}$ NMR $(100 \mathrm{MHz}): \delta$ $190.24,179.51,145.73,143.46,143.11142 .70,142.24,141.41,134.98,132.02,131.65$, $128.34,119.67,117.69,34.66,31.35,30.21,28.23,26.33, \mathrm{~ms}(\mathrm{ESI}+): \mathrm{m} / z: 428.11[\mathrm{M}+\mathrm{H}]+$. Mol.Formula: $\mathrm{C}_{25} \mathrm{H}_{24} \mathrm{~N}_{4} \mathrm{OS}$.

4-(3-(4-Fluorophenyl)-1-phenyl-1H-pyrazol-4-yl)-7,7-dimethyl-2-thioxo1,2,3,4,7,8-hexahydroquinazolin-5(6H)-one $(\mathbf{4 k})$

Milky White solid, 93\%yield, mp-173 ${ }^{\circ} \mathrm{C}$; IR(KBr): 3254,1749,1639 ${ }^{1} \mathrm{H}$ NMR (DMSO$\left.\mathrm{d}_{6}, 400 \mathrm{MHz}\right) \delta 7.82(\mathrm{~d}, 2 \mathrm{H}, \mathrm{J}=10.0 \mathrm{~Hz}) \delta 6.79-6.88(\mathrm{~m}, 4 \mathrm{H}) \delta 6.48-7.55(\mathrm{~m}, 5 \mathrm{H}) \delta 5.34(\mathrm{~s}, 1 \mathrm{H})$ $\delta 4.79(\mathrm{~s}, 1 \mathrm{H}) \delta 2.29-2.33(\mathrm{~d}, 2 \mathrm{H}, \mathrm{J}=16 \mathrm{~Hz}) \delta 1.14(\mathrm{~s}, 3 \mathrm{H}) \quad \delta 1.04(\mathrm{~s}, 3 \mathrm{H}){ }^{13} \mathrm{CNMR}(100 \mathrm{MHz})$ : $\delta 193.45,181.63,147.64,144.77,144.12143 .65,142.96,142.14,137.39,132.97,132.83$, $130.76,120.36,118.61,35.34,33.84,31.65,29.12,27.67, \mathrm{~ms}(\mathrm{ESI}+): \mathrm{m} / z: 445.57[\mathrm{M}+\mathrm{H}]+$. Mol.Formula: $\mathrm{C}_{25} \mathrm{H}_{23} \mathrm{FN}_{4} \mathrm{OS}$.

\section{4-(3-(4-Chlorophenyl)-1-phenyl-1H-pyrazol-4-yl)-7,7-dimethyl-2-thioxo- 1,2,3,4,7,8-hexahydroquinazolin-5(6H)-one (4l)}

Milky White solid, 92\% yield, mp-221 ${ }^{\circ} \mathrm{C}$; IR (KBr): 3254,1723,1651 ${ }^{1} \mathrm{H}$ NMR (DMSO$\left.\mathrm{d}_{6}, 400 \mathrm{MHz}\right) \delta 7.66(\mathrm{~d}, 2 \mathrm{H}, \mathrm{J}=12.0 \mathrm{~Hz}) \quad \delta 6.69-6.76(\mathrm{~m}, 4 \mathrm{H}) \delta 6.56-7.64(\mathrm{~m}, 5 \mathrm{H}) \delta 5.12(\mathrm{~s}, 1 \mathrm{H})$ $\delta 4.72(\mathrm{~s}, 1 \mathrm{H}) \delta 2.21-2.25(\mathrm{~d}, 2 \mathrm{H}, \mathrm{J}=16 \mathrm{~Hz}) \delta 1.12(\mathrm{~s}, 3 \mathrm{H}) \quad \delta 1.01(\mathrm{~s}, 3 \mathrm{H}){ }^{13} \mathrm{CNMR}(100 \mathrm{MHz})$ : $\delta 192.89,181.43,146.72,144.73,143.36,142.94,142.67,141.85,135.43,131.64,131.64$, $129.63,122.58,119.38,33.96,33.14,29.34,28.78,27.31, \mathrm{~ms}(\mathrm{ESI}+): \mathrm{m} / z: 462.82[\mathrm{M}+\mathrm{H}]+$. Mol.Formula: $\mathrm{C}_{25} \mathrm{H}_{19} \mathrm{ClN}_{4} \mathrm{OS}$.

\section{Conclusion}

In present methodology water was using as a solvent due to low toxicity, no inflammability, and including its low cost. We successfully synthesized octahydroquinazoline-2(1H)-thiones and octahydroquinazolin-2 $(1 H)$-ones derivatives with excellent yields by one pot reaction of pyrazole containing aldehydes with dimedone and thiourea or urea in the presence of catalytic amount of $p$ - $\mathrm{TsOH}$ in water. 


\section{Acknowledgment}

The authors are grateful to The Head, Department of Chemistry Dr. H. S. Gour University Sagar for providing necessary facilities to carry out this work. We are grateful to The Director, SAIF, Panjab University for providing spectral data.

\section{References}

1. Desai N C and Dodiya A M, Arab J Chem., 2014,6(6), 906-913; DOI:10.1016/j.arabjc.2011.08.007

2. Tobe M, Isobe Y, Tomizawa H, Nagasaki T, Obara F and Hayashi H, Bioorg Med Chem., 2003, 11(4), 609-616; DOI:10.1016/S0968-0896(02)00338-3

3. Guang-Fang X, Bao-An S, Pinaki S B, Song Y, Pei-Quan Z, Lin-Hong J, Wei X, DeYu H and Ping L, Bioorg Med Chem., 2007, 15, 3768-3774; DOI:10.1016/j.bmc.2007.03.037

4. Al-Saadi M S, Faidallah H M and Rostom S A F, Arch Pharm Chem Life Sci., 2008, 341(7), 424-434; DOI:10.1002/ardp.200800026

5. Wakelin I, Waring M J, DNA Intercalating Agents. In: Sammes, P.G. (Ed.), In: Comp Med Chem, vol. 2. Pergamon, Oxford, UK, 1990, 725.

6. $\quad$ Bondock S, Khalifa W and Fadda A A, Eur J Med Chem., 2007, 42(7), 948-954; DOI:10.1016/j.ejmech.2006.12.025

7. Yarim M, Sarac S, Ertan M, Kilic F S and Erol K, Arzneim-Forsch, 2002, 27, 52.

8. Yarim M, Sarac S, Kilic F S and Erol K, Il Farmaco, 2003, 58(1), 17-24; DOI:10.1016/S0014-827X(02)00009-5

9. Hulme C, Chappeta S, Griffith C, Lee Y S and Dietrich J, Tetrahedron Lett., 2009, 26(16), 1939-1942; DOI:10.1016/S0040-4039(00)98346-1

10. Ruijter E, Scheffelaar R and Orru R V A, Angew Chem Int Ed., 2011, 50(28), 63246327; DOI:10.1002/anie.201100821

11. Hassani Z, Islami M R and Kalantari M, Bioorg Med Chem Lett., 2006, 16(17), 44794482; DOI:10.1016/j.bmcl.2006.06.038

12. Kirti S Niralwad, Bapurao B Shingate, Murlidhar S Shingare, Tetrahedron Lett., 2010, 51(28), 3616-3618; DOI:10.1016/j.tetlet.2010.04.118

13. Niralwad K S, Shingate B B and Shingare M S, J Chin Chem Soc., 2010, 57(1), 8992; DOI:10.1002/jccs.201000014

14. Khurana J M, Kumar S Monatsh, Monatshefte für Chemie-Chemical Monthly.,2010, 141(5), 561-564; DOI:10.1007/s00706-010-0306-4

15. Anastasiya Yu Andriushchenko, Vyacheslav E Saraev, Svetlana V Shishkina, Oleg V Shishkin, Vladimir I Musatov, Sergey M Desenko and Valentin A Chebanov, ARKIVOC, 2013, 3, 61-80.

16. Jianjian W, Wenjie X, Jiawen R, Xiaohui L, Guanzhong L and Yanqin W, Green Chem., 2011, 13, 2678-2681; DOI:10.1039/C1GC15306D 